\title{
Synthetic studies on some 3-[(5-arylidene-4-oxo-1,3-thiazolidin-2- yliden)amino]-2-phenylquinazolin-4(3H)-ones and their ethoxyphthalimide derivatives
}

\author{
Usha Ameta, Swati Ojha, Dinesh Bhambi, and Ganpat L. Talesara* \\ Synthetic Organic Chemistry Laboratory, Department of Chemistry, M.L. Sukhadia University, \\ Udaipur (Raj.) - 313001, India \\ E-mail: gtalesara@yahoo.com
}

\begin{abstract}
A simple method for the synthesis of title compounds is reported, which were isolated from a series of reactions. After a nucleophilic reaction of 2-phenyl-3,1-benzoxazin-4(3H)-one (1) with thiosemicarbazide to furnish quinazolinylthiourea (2), followed by cyclisation with chloroacetic acid, 3-[(4-oxo-1,3-thiazolidin-2-yliden)amino]-2-phenylquinazolin-4(3H)-one (3) was yielded, which was converted to corresponding arylidene derivatives (5a-f) by treatment with various aldehydes (4a-f). Subsequent condensation of (5a-f) with phthalimidoxyethylbromide gave title compounds (7a-f). The structure of isolated compounds has been determined by means of IR, ${ }^{1} \mathrm{H}$ NMR and mass spectroscopy.
\end{abstract}

Keywords: Benzoxazin-4(3H)-one, quinazolinylthiourea, arylidene, quinazolin-4(3H)-one, cyclisation, condensation.

\section{Introduction}

Quinazolone derivatives have evoked considerable attention in recent years as these are endowed with wide range of pharmaceutical activities. $3 \mathrm{H}-$ Quinazolin-4-one represents a useful nucleus for preparation of some new sedative/ hypnotic and anticonvulsant agents, since such a heterocyclic system possesses the pharmacophoric moiety ${ }^{1}$. Of the various quinazolines reported, the C-2 and N-3 disubstituted quinazolines exhibited interesting pharmacological activities like analgesic, anti-inflammatory ${ }^{2}$, antibacterial ${ }^{3}$, anticonvulsant ${ }^{4}$ etc. 2,3-Disubstituted quinazolones have been demonstrated to be associated with potent antiviral and antihypertensive acitivities $^{5}$. Characterization of potential NMDA and cholecystokinin antagonist II-lipophilicity studies on quinazolones are also documented ${ }^{6}$.

Furthermore, 4-thiazolidinones have been synthesized ${ }^{7}$ and used for the treatment of $\operatorname{cardiac}^{8}$ diseases. Modifications on 2,3,4 and 5 positions of 4-thiazolidinone give out antidiabetic 
drugs and potent aldose reductase inhibitors, which are used in the treatment of diabetic complications like catracts, nephropathy, neuropathy ${ }^{9}$. 4-Substituted thiazolidinone derivatives have been synthesized and reported to show a variety of pharmacological and microbiological activities such as antibacterial ${ }^{10}$, antifungal, analgesic etc. Significant antiparkinsonian activity against tremor, rigidity, hypokinesia and catatonia has been evaluated "in vivo" in rats and mice in quinazolinylthiazolidinone ${ }^{11}$. Anticonvulsant ${ }^{12}$ and anticancer ${ }^{13}$ activities have been observed in many alkoxyphthalimide derivatives. Many amino-oxy compounds have been tested for their ability to inhibit the growth of the malaria parasite Plasmodium falciparum in vitro ${ }^{14}$. Heterocyclic rings attached to alkoxyphthalimide group have been synthesized ${ }^{15}$ and tested for antimicrobial and antimalarial ${ }^{16}$ activities.

Led by above facts, coupled with our desire of synthesizing alkoxyphthalimide derivatives, we report herein the synthesis of some new heterocycles incorporating above moieties together in order to prepare the molecules having enhanced biological activity.

\section{Results and Discussion}

In the present work, an attempt has been made to undertake the synthesis of ethoxyphthalimide derivatives of some thiazolidinoquinazolinones through a multi step process. For this purpose, the required 2-phenyl-3,1-benzoxazin-4(3H)-one (1) was prepared by cyclisation reaction between anthranilic acid and benzoyl chloride using pyridine as a solvent and also a base. Formation of the product was confirmed by a sharp band at $1720 \mathrm{~cm}^{-1}$ for $\mathrm{C}=\mathrm{O}$ group along with a peak at $1180 \mathrm{~cm}^{-1}$ for $\mathrm{C}-\mathrm{O}$ stretching in IR spectra. Benzoxazine (1) was converted to quinazolinylthiourea (2) by its nucleophilic substitution reaction with thiosemicarbazide. Insertion of nitrogen in the ring was characterized by disappearance of band at $1180 \mathrm{~cm}^{-1}$ of C-O and shift of carbonyl band from 1720 to $1692 \mathrm{~cm}^{-1}$. Appearance of new peaks near 3400 and $3300 \mathrm{~cm}^{-1}$ for N-H stretching also helped in assigning structure of (2). When (2) was treated with chloroacetic acid in presence of sodium acetate as a base, nucleophilic reaction took place at the thiourea site of molecule and thiazolidinone ring was formed as a result, to yield a new heterocyclic compound (3) having two free different heterocyclic rings namely quinazolone and thiazolidinone in it. New band in IR at $2983 \mathrm{~cm}^{-1}$ and a singlet in NMR at $\delta 3.5$ for methylene protons and a singlet at $\delta 5.3$ for $\mathrm{NH}$ proton were in accordance with the structure of thiazolidinone ring. Its condensation with different aldehydes (4a-f) afforded corresponding arylidene derivatives (5a-f) in quantitative yield.

Formation of (5a) was confirmed by appearance of carbonyl peak at $1665 \mathrm{~cm}^{-1}$, which is due to $\alpha, \beta$-unsaturated carbonyl group. Disappearance of singlet at $\delta 3.5$ (active $\mathrm{CH}_{2}$ ) and appearance of new singlet at $\delta 6.0(\mathrm{C}=\mathrm{CH}-\mathrm{Ar})$ also confirm its formation.

Hydrogen of 'N-H' in thiazolidinone ring was replaced by ethoxyphthalimide group by treating (5a) with phthalimidoxyethylbromide (6) using pyridine as a base to furnish 3-[(3- $N$ ethoxyphthalimido-5-(4'-methoxybenzylidene)-4-oxo-1,3-thiazolidin-2-yliden)amino]-2- 
phenylquinazolin-4(3H)-one (7a). Disappearance of $\mathrm{N}-\mathrm{H}$ stretching band at $3335 \mathrm{~cm}^{-1}$ as in (5a) and appearance of new bands at 1380 and $1180 \mathrm{~cm}^{-1}$ for $\mathrm{N}-\mathrm{O}$ and $\mathrm{C}-\mathrm{O}$ stretching respectively confirmed synthesis of final compound (7a).

\section{Experimental Section}

General Procedure. Melting points of all synthesized compounds were taken in open capillaries and are uncorrected. IR spectra $(\mathrm{KBr})$ were recorded on a Perkin- Elmer 1300 FT IR spectrometer and ${ }^{1} \mathrm{H}$ NMR were determined on a Bruker WM-400 (400 MHz FT NMR) spectrometer using TMS as internal standard. Mass spectra were recorded on Jeol D-300 (EI) and Jeol Sx-102 (FAB) spectrometer. Purity of compounds was checked by elemental analysis and TLC using silica gel-' $G$ ' as adsorbent and visualization was accomplished with iodine. Compound (6) was synthesized as given in literature methods ${ }^{17}$.

Synthesis of 2-phenyl-3,1-benzoxazin-4(3H)-one (1). To a stirred solution of anthranilic acid $(0.01 \mathrm{~mole})$ in pyridine $(60 \mathrm{~mL})$, benzoyl chloride $(0.01 \mathrm{~mole})$ was added dropwise, maintaining the temperature near $8^{\circ} \mathrm{C}$ for 1 hour. Reaction mixture was stirred for another 2 hours at room temperature. While stirring a solid product separates out. Whole reaction mixture was neutralized with $\mathrm{NaHCO}_{3}$ solution. A pale yellow solid deposited which was filtered, washed with water and recrystallised from ethanol. 1: yield $78 \%$, m.p. $114^{\circ} \mathrm{C}$; IR $(\mathrm{KBr}) \mathrm{cm}^{-1}$ : 3030 (C-H, Ar-H), $1720(\mathrm{C}=\mathrm{O}), 1590(\mathrm{C}=\mathrm{N}), 1180(\mathrm{C}-\mathrm{O}) ;{ }^{1} \mathrm{H}$ NMR (DMSO-d $) \delta 7.6(\mathrm{~m}, 9 \mathrm{H}$, Ar-H); Anal. Calcd. for $\mathrm{C}_{14} \mathrm{H}_{9} \mathrm{NO}_{2}$ : C, 75.33; $\mathrm{H}, 4.03$; N, 6.27. Found: C, 75.15; H, 4.00; , $6.25 \%$.

Synthesis of $N^{\prime}$-[2-phenyl-4(3H)-oxo-quinazolin-3-yl]thiourea (2). Compound 1 (0.01 mole) was dissolved in ethanol and thiosemicarbazide $(0.01$ mole $)$ in ethanol was added to it with a catalytic amount of pyridine. Reaction mixture was refluxed for 4 hours and after cooling a crystalline product was obtained. It was filtered and recrystallised from ethanol to yield needle shaped shining white crystals. 2: yield $70 \%$, m.p. $180{ }^{\circ} \mathrm{C}$; IR $(\mathrm{KBr}) \mathrm{cm}^{-1}: 3418,3340(\mathrm{~N}-\mathrm{H})$, $3028(\mathrm{C}-\mathrm{H}, \mathrm{Ar}-\mathrm{H}), 1692(\mathrm{C}=\mathrm{O}), 1585(\mathrm{C}=\mathrm{N}), 1270(\mathrm{C}=\mathrm{S}) ;{ }^{1} \mathrm{H}$ NMR $\left(\mathrm{DMSO}^{-} \mathrm{d}_{6}\right): \delta 8.3(\mathrm{~m}, 3 \mathrm{H}$, NH.C $=$ S.NH $\left.\mathrm{NH}_{2}\right), 7.8(\mathrm{~m}, 9 \mathrm{H}, \mathrm{Ar}-\mathrm{H})$; Anal. Calcd. for $\mathrm{C}_{15} \mathrm{H}_{12} \mathrm{~N}_{4} \mathrm{OS}: \mathrm{C}, 60.81 ; \mathrm{H}, 4.05 ; \mathrm{N}, 18.91$. Found: C, 60.56; H, 4.02; N, 18.72\%.

Synthesis of 3-[(4-oxo-1,3-thiazolidin-2-yliden)amino]-2-phenylquinazolin-4(3H)-one (3). A mixture of 2 (0.01 mole), chloroacetic acid (0.01 mole) and anhydrous sodium acetate (0.02 mole) in absolute alcohol was refluxed for $12 \mathrm{hrs}$. Excess of solvent was distilled off and the reaction mixture was poured on crushed ice. The solid obtained was filtered, washed with water and recrystallised from DMF to get white crystals. 3: yield $71 \%$, m.p. $260{ }^{\circ} \mathrm{C}$; IR ( $\left.\mathrm{KBr}\right) \mathrm{cm}^{-1}$ : 3350 (N-H), 3025 (C-H, Ar-H), 2983 (C-H, CH ), 1690, 1671 (C=O), 698 (C-S-C); ${ }^{1} \mathrm{H}$ NMR $\left(\mathrm{DMSO}_{\mathrm{d}}\right): \delta 7.6(\mathrm{~m}, 9 \mathrm{H}, \mathrm{Ar}-\mathrm{H}), 5.3(\mathrm{~s}, 1 \mathrm{H}, \mathrm{NH}), 3.5\left(\mathrm{~s}, 2 \mathrm{H}, \mathrm{CH}_{2}\right)$; Anal. Calcd. for $\mathrm{C}_{17} \mathrm{H}_{12} \mathrm{~N}_{4} \mathrm{O}_{2} \mathrm{~S}: \mathrm{C}, 60.71 ; \mathrm{H}, 3.57$; N, 16.66. Found: C, 60.56; H, 3.56; N, 16.42\%. 


\section{Reaction Scheme}

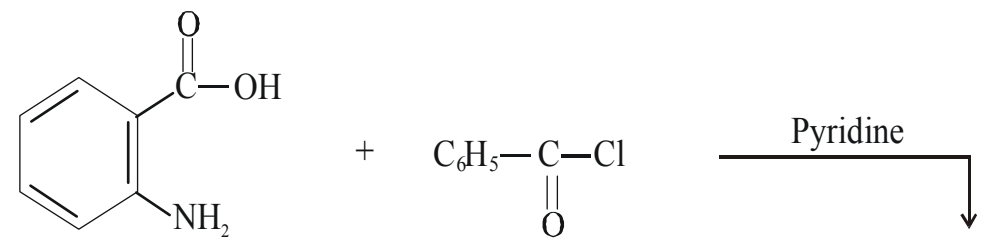

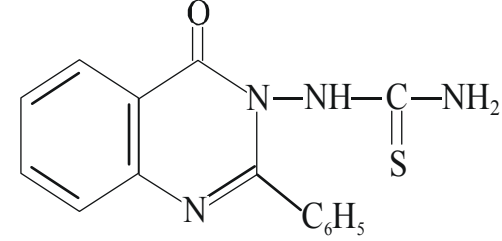

(2)

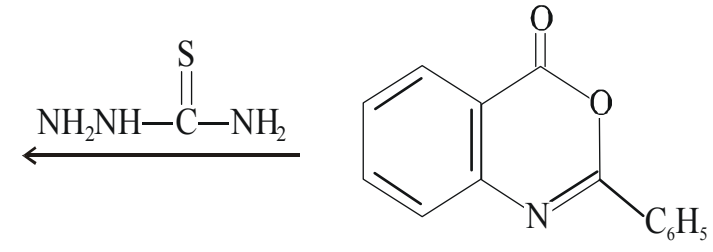

(1)<smiles>CCCCCCc1nc2ccccc2c(=O)n1N=C1NNC(=O)CS1</smiles>

(3)

4

a

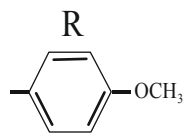

b<smiles>Cc1ccccc1</smiles>

c<smiles>C[Si](C)(C)c1ccc(I)cc1</smiles>

d

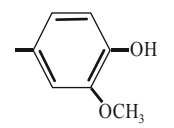

e<smiles>Clc1ccc(I)cc1</smiles>

f<smiles>[R]CON1C(=O)c2ccccc2C1=O</smiles>

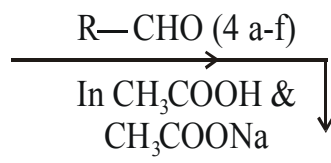<smiles>[R]C=C1SC(=Nn2c(CC)nc3ccccc3c2=O)NC1=O</smiles>

(5 a-f)

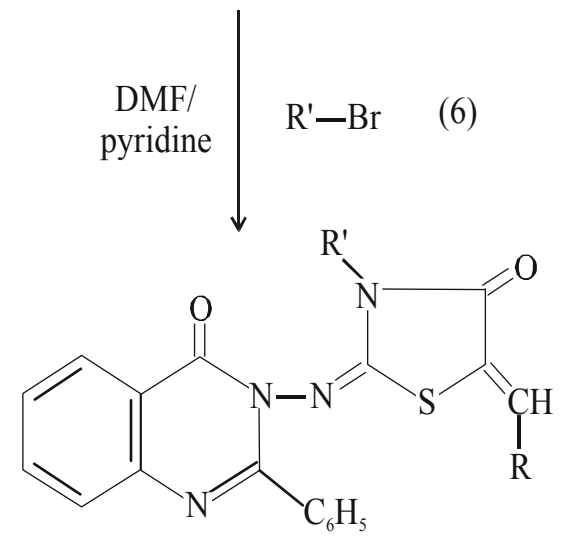

(7 a-f) 
Synthesis of 3-[(5-(4'-methoxybenzylidene)-4-oxo-1,3-thiazolidin-2-yliden)amino]-2phenylquinazolin-4(3H)-one (5a). An equimolar mixture of 3 (0.01 mole) and anisaldehyde $4 \mathbf{a}$ $(0.01$ mole $)$ in glacial acetic acid was taken in a round bottom flask and anhydrous sodium acetate $(0.02$ mole) was added as base and refluxed for 8 hours. Reaction mixture was allowed to cool and the solid separated was recrystallised from DMF 5a: yield 69\%, m.p. $260{ }^{\circ} \mathrm{C}$; IR (KBr) $\mathrm{cm}^{-1}$ : $3335(\mathrm{~N}-\mathrm{H}), 3024(\mathrm{C}-\mathrm{H}, \mathrm{Ar}-\mathrm{H}), 2980\left(\mathrm{C}-\mathrm{H}, \mathrm{CH}_{3}\right), 1687,1665(\mathrm{C}=\mathrm{O}), 1580(\mathrm{C}=\mathrm{N}), 1175$ (C-O), $690(\mathrm{C}-\mathrm{S}-\mathrm{C}) ;{ }^{1} \mathrm{H}$ NMR $\left(\mathrm{DMSO}_{6}\right): \delta$ 7.3-7.8 (m, 13H, Ar-H), $6.0(\mathrm{~s}, 1 \mathrm{H},=\mathrm{CH}), 5.1(\mathrm{~s}$, $1 \mathrm{H}, \mathrm{NH}), 3.8\left(\mathrm{~s}, 3 \mathrm{H}, \mathrm{OCH}_{3}\right)$, Anal. Calcd. for $\mathrm{C}_{25} \mathrm{H}_{18} \mathrm{~N}_{4} \mathrm{O}_{3} \mathrm{~S}: \mathrm{C}, 66.07 ; \mathrm{H}, 3.96 ; \mathrm{N}, 12.33$. Found: C, 65.94; H, 3.82; N, 12.22\%.

Compounds $\mathbf{5 b - f}$ were also prepared in a similar way with minor modifications in reflux time. Their characteristic spectral \& analytical data are given below:

3-[(5-(4'-Methoxybenzylidene)-4-oxo-1,3-thiazolidin-2-yliden)amino]-2-phenylquinazolin4(3H)-one (5b). Yield 67\%, m.p. $250^{\circ} \mathrm{C}$; IR (KBr) cm ${ }^{-1}: 3337$ (N-H), 3025 (C-H, Ar-H), 1687, $1668(\mathrm{C}=\mathrm{O}), 1580(\mathrm{C}=\mathrm{N}), 692(\mathrm{C}-\mathrm{S}-\mathrm{C}) ;{ }^{1} \mathrm{H}$ NMR $\left(\mathrm{DMSO}^{-} \mathrm{d}_{6}\right): \delta 7.3-7.8(\mathrm{~m}, 14 \mathrm{H}, \mathrm{Ar}-\mathrm{H}), 6.1(\mathrm{~s}$, $1 \mathrm{H},=\mathrm{CH}), 5.1(\mathrm{~s}, 1 \mathrm{H}, \mathrm{NH})$ : Anal. Calcd. for $\mathrm{C}_{24} \mathrm{H}_{16} \mathrm{~N}_{4} \mathrm{O}_{2} \mathrm{~S}: \mathrm{C}, 67.92 ; \mathrm{H}, 3.77 ; \mathrm{N}, 13.20$. Found: C, 67.84; H, 3.49; N, $13.04 \%$.

3-[(5-(4'-Dimethylaminobenzylidene)-4-oxo-1,3-thiazolidin-2-yliden)amino]-2phenylquinazolin-4(3H)-one (5c). Yield $64 \%$, m.p. $>300{ }^{\circ} \mathrm{C}$; IR (KBr) cm ${ }^{-1}: 3330(\mathrm{~N}-\mathrm{H})$, $3021\left(\mathrm{C}-\mathrm{H}, \quad\right.$ Ar-H), 2981, $2890\left(\mathrm{C}-\mathrm{H}, \mathrm{CH}_{3}\right), 1685,1663(\mathrm{C}=\mathrm{O}) ;{ }^{1} \mathrm{H}$ NMR (DMSO-d $)$; $\delta$ 7.2-7.9 $(\mathrm{m}, 13 \mathrm{H}, \mathrm{Ar}-\mathrm{H}), 5.9(\mathrm{~s}, 1 \mathrm{H},=\mathrm{CH}), 2.9\left(\mathrm{~s}, 6 \mathrm{H}, \mathrm{N}\left(\mathrm{CH}_{3}\right)_{2}\right)$; Anal. Calcd. for $\mathrm{C}_{26} \mathrm{H}_{21} \mathrm{~N}_{5} \mathrm{O}_{2} \mathrm{~S}: \mathrm{C}$, 66.80; H, 4.49; N, 14.98. Found : C, 66.42; H, 4.32; N, 14.72\%.

3-[(5-(4'-Hydroxy-3'-methoxybenzylidene)-4-oxo-1,3-thiazolidin-2-yliden)amino]-2-

phenylquinazolin-4(3H)-one (5d). Yield $64 \%$, m.p.> $300{ }^{\circ} \mathrm{C}$; IR (KBr) cm ${ }^{-1}: 3400(\mathrm{br}, \mathrm{OH})$, 3325 (N-H), 3018 (C-H, Ar-H), 1684, 1661 (C=O), 1180 (C-O); ${ }^{1} \mathrm{H}$ NMR (DMSO-d $)$ ); $\delta$ 7.2-7.9 $(\mathrm{m}, 12 \mathrm{H}, \mathrm{Ar}-\mathrm{H}), 5.7(\mathrm{~s}, 1 \mathrm{H},=\mathrm{CH}), 3.8\left(\mathrm{~s}, 3 \mathrm{H}, \mathrm{OCH}_{3}\right)$; Anal. Calcd. for $\mathrm{C}_{25} \mathrm{H}_{18} \mathrm{~N}_{4} \mathrm{O}_{4} \mathrm{~S}: \mathrm{C}, 63.82$; H, 3.82; N, 11.91. Found: C, 63.76; H, 3.48; N, 11.49\%.

3-[(5-(4'-Chlorobenzylidene)-4-oxo-1,3-thiazolidin-2-yliden)amino]-2-phenylquinazolin4(3H)-one (5e). Yield 70 \%, m.p. $288^{\circ} \mathrm{C}$; IR (KBr) cm ${ }^{-1}: 3318(\mathrm{~N}-\mathrm{H}), 3018$ (C-H, Ar-H), 3015 (C-H, Ar-H), $748(\mathrm{C}-\mathrm{Cl}) ;{ }^{1} \mathrm{H}$ NMR $\left(\mathrm{DMSO}_{-} \mathrm{d}_{6}\right) ; \delta$ 7.1-7.6 (m, 13H, Ar-H), $5.8(\mathrm{~s}, 1 \mathrm{H},=\mathrm{CH})$; Anal. Calcd. for $\mathrm{C}_{24} \mathrm{H}_{15} \mathrm{~N}_{4} \mathrm{O}_{2} \mathrm{SCl}: \mathrm{C}, 62.88 ; \mathrm{H}, 3.27 ; \mathrm{N}, 12.22$. Found: C, 62.69; H, 3.13; N, $12.04 \%$.

3-[(5-(2-Furyl)methylene-4-oxo-1,3-thiazolidin-2-yliden)amino]-2-phenylquinazolin-4(3H)one (5f). Yield $60 \%$, m.p. $248^{\circ} \mathrm{C}$; IR (KBr) cm ${ }^{-1}$ : $3322(\mathrm{~N}-\mathrm{H}), 3024$ (C-H, Ar-H), 1180 (C-OC), ${ }^{1} \mathrm{H}$ NMR $\left(D_{M S O}-d_{6}\right) ; \delta$ 6.9-7.5 $(\mathrm{m}, 12 \mathrm{H}, \mathrm{Ar}-\mathrm{H}), 5.9(\mathrm{~s}, 1 \mathrm{H},=\mathrm{CH})$; Anal. Calcd. for $\mathrm{C}_{22} \mathrm{H}_{14} \mathrm{~N}_{4} \mathrm{O}_{3} \mathrm{~S}: \mathrm{C}, 63.76 ; \mathrm{H}, 3.38 ; \mathrm{N}, 13.52$. Found : C, 63.49; H, 3.32; N, 13.42\%.

Synthesis of 3-[(3- $N$-ethoxyphthalimido-5-(4'-methoxybenzylidene)-4-oxo-1,3-thiazolidin-2yliden)amino]-2-phenylquinazolin-4(3H)-one (7a). Phthalimidoxyethyl bromide 6 (0.01 mole) and 5a (0.01 mole) were dissolved in DMF and pyridine (0.02 mole) was added as base. Reaction mixture was heated to reflux for 22 hours and resulting mixture was cooled and the solid obtained was filtered, washed, dried and recrystallised from DMF to yield title 
compound.7a. Yield 62\%, m.p. $120{ }^{\circ} \mathrm{C}$; IR (KBr) cm ${ }^{-1}: 3030(\mathrm{C}-\mathrm{H}, \mathrm{Ar}-\mathrm{H}), 2970,2880(\mathrm{C}-\mathrm{H}$, $\left.\mathrm{CH}_{2}\right)$, 1710, 1685, $1662(\mathrm{C}=\mathrm{O}), 1585(\mathrm{C}=\mathrm{N}), 1380(\mathrm{~N}-\mathrm{O}), 1180(\mathrm{C}-\mathrm{O}), 692(\mathrm{C}-\mathrm{S}-\mathrm{C}) ;{ }^{1} \mathrm{H}$ NMR $\left(\right.$ DMSO-d $\left._{6}\right): \delta 7.77(\mathrm{~m}, 17 \mathrm{H}, \mathrm{Ar}-\mathrm{H}), 6.2(\mathrm{~s}, 1 \mathrm{H},=\mathrm{CH}), 3.7\left(\mathrm{~s}, 3 \mathrm{H}, \mathrm{OCH}_{3}\right), 3.3\left(\mathrm{t}, 2 \mathrm{H}, \mathrm{OCH}_{2}\right), 2.8$ $\left(\mathrm{t}, 2 \mathrm{H}, \mathrm{NCH}_{2}\right) ; \mathrm{MS}: \mathrm{m} / \mathrm{z}: 643\left[\mathrm{M}^{+}\right], 536,455,390,221,132,107$. Anal. Calcd. for $\mathrm{C}_{35} \mathrm{H}_{25} \mathrm{~N}_{5} \mathrm{O}_{6} \mathrm{~S}:$ C, $65.31 ; \mathrm{H}, 3.88 ; \mathrm{N}, 10.88$. Found : C, 65.20; H, 3.76; N, 10.48\%.

Compounds (7b-f) were also synthesized using appropriate reagents and by small changes in reflux time. Their characteristic physical \& spectral data are given below:

3-[(3- $N$-Ethoxyphthalimido-5-benzylidene-4-oxo-1,3-thiazolidin-2-yliden)amino]-2phenylquinazolin-4(3H)-one (7b). Yield 59\%, m.p. $108{ }^{\circ} \mathrm{C}$; IR (KBr) cm ${ }^{-1}: 3034(\mathrm{C}-\mathrm{H}, \mathrm{Ar}-\mathrm{H})$, 2975, $2880\left(\mathrm{C}-\mathrm{H}, \mathrm{CH}_{2}\right)$, 1712, 1688, $1662(\mathrm{C}=\mathrm{O}), 1380(\mathrm{~N}-\mathrm{O}) 1182(\mathrm{C}-\mathrm{O})$ ) ${ }^{1} \mathrm{H}$ NMR (DMSO$\left.\mathrm{d}_{6}\right): \delta 7.78(\mathrm{~m}, 18 \mathrm{H}, \mathrm{Ar}-\mathrm{H}), 6.3(\mathrm{~s}, 1 \mathrm{H},=\mathrm{CH}), 3.3\left(\mathrm{t}, 2 \mathrm{H}, \mathrm{OCH}_{2}\right), 2.9\left(\mathrm{t}, 2 \mathrm{H}, \mathrm{NCH}_{2}\right) ; \mathrm{MS}: \mathrm{m} / \mathrm{z}$ : $613\left[\mathrm{M}^{+}\right]$, 423, 364, 132, 77 .Anal. Calcd. for $\mathrm{C}_{34} \mathrm{H}_{23} \mathrm{~N}_{5} \mathrm{O}_{6} \mathrm{~S}: \mathrm{C}, 66.55 ; \mathrm{H}, 3.75 ; \mathrm{N}, 11.41$. Found: C, 66.34; H, 3.61; N, 11.29\%.

3-[(3- $N$-Ethoxyphthalimido-5-(4-dimethylaminobenzylidene)-4-oxo-1,3-thiazolidin-2yliden)amino]-2-phenylquinazolin-4(3H)-one (7c). Yield $58 \%$, m.p. $136^{\circ} \mathrm{C}$; $\mathrm{IR}(\mathrm{KBr}) \mathrm{cm}^{-1}$ : 3028 (C-H, Ar-H), 2970, 2880 (C-H, CH $),$ 1708, 1685, 1659 (C=O, 1378 (N-O), 1182 (C-O); ${ }^{1} \mathrm{H}$ NMR (DMSO-d 6 ): $\delta$ 7.2-7.7 (m, 17H, Ar-H), $6.2(\mathrm{~s}, 1 \mathrm{H},=\mathrm{CH}), 3.2\left(\mathrm{t}, 2 \mathrm{H}, \mathrm{OCH}_{2}\right), 2.9(\mathrm{t}, 2 \mathrm{H}$, $\left.\mathrm{NCH}_{2}\right) ; \mathrm{MS}: \mathrm{m} / \mathrm{z}: 654\left[\mathrm{M}^{+}\right], 466,433,221,188,132,120$. Anal. Calcd. for $\mathrm{C}_{36} \mathrm{H}_{26} \mathrm{~N}_{5} \mathrm{O}_{6} \mathrm{~S}: \mathrm{C}$, $66.05 ; \mathrm{H}, 3.97 ; \mathrm{N}, 10.70$. Found : C, 65.94; H, 3.82; N, 10.61\%.

3-[(3- $N$-Ethoxyphthalimido-5-(4'-hydroxy-3'-methoxybenzylidene)-4-oxo-1,3-thiazolidin-2yliden)amino]-2-phenylquinazolin-4(3H)-one (7d). Yield 54\%, m.p. $148{ }^{\circ} \mathrm{C}$; IR $(\mathrm{KBr}) \mathrm{cm}^{-1}$ : 3400 (br, OH), 3030 (C-H, Ar-H), 1370 (N-O), 1175 (C-O); ${ }^{1} \mathrm{H}$ NMR (DMSO-d 6 ): $\delta$ 7.1-7.8 (m, 16H, Ar-H), $6.1(\mathrm{~s}, 1 \mathrm{H},=\mathrm{CH}), 3.8\left(\mathrm{~s}, 1 \mathrm{H}, \mathrm{OCH}_{3}\right), 3.1\left(\mathrm{t}, 2 \mathrm{H}, \mathrm{OCH}_{2}\right) ; 2.8\left(\mathrm{t}, 2 \mathrm{H}, \mathrm{NCH}_{2}\right) ; \mathrm{MS}$ : $\mathrm{m} / \mathrm{z}: 659\left[\mathrm{M}^{+}\right], 469,438,190,123$. Anal. Calcd. for $\mathrm{C}_{35} \mathrm{H}_{25} \mathrm{~N}_{5} \mathrm{O}_{7} \mathrm{~S}: \mathrm{C}, 63.73 ; \mathrm{H}, 3.79 ; \mathrm{N}, 12.74$. Found : C, 63.54; H, 3.77; N, 12.66\%.

3-[(3- $\mathrm{N}$-Ethoxyphthalimido-5-(4'-chlorobenzylidene)-4-oxo-1,3-thiazolidin-2-yliden)amino]2-phenylquinazolin-4(3H)-one (7e). Yield 61\%, m.p. $132{ }^{\circ} \mathrm{C}$; IR $(\mathrm{KBr}) \mathrm{cm}^{-1}: 3025(\mathrm{C}-\mathrm{H}, \quad \mathrm{Ar}-$ $\mathrm{H}), 2965,2870\left(\mathrm{C}-\mathrm{H}, \mathrm{CH}_{2}\right)$, 1700, 1680, $1660(\mathrm{C}=\mathrm{O}), 1580(\mathrm{C}=\mathrm{N}), 1370(\mathrm{~N}-\mathrm{O}), 1170(\mathrm{C}-\mathrm{O}), 746$ (C-Cl); ${ }^{1} \mathrm{H}$ NMR (DMSO-d $\left.{ }_{6}\right): \delta$ 7.2-7.6 (m, 17H, Ar-H), $6.0(\mathrm{~s}, 1 \mathrm{H},=\mathrm{CH}), 3.2\left(\mathrm{t}, 2 \mathrm{H}, \mathrm{OCH}_{2}\right) ; 2.7$ (t, $2 \mathrm{H}, \mathrm{NCH}_{2}$ ); MS : m/z : $649\left[\mathrm{M}+2^{+}\right], 647\left[\mathrm{M}^{+}\right], 457,428,426,200,192,113,111$. Anal. Calcd. for $\mathrm{C}_{34} \mathrm{H}_{22} \mathrm{~N}_{5} \mathrm{O}_{5} \mathrm{~S}$ : C, 63.06; H, 3.40; N, 10.81. Found : C, 62.88; H, 3.32; N, 10.72\%.

3-[(3- $\mathrm{N}$-Ethoxyphthalimido-5-(4'-furylmethylene)-4-oxo-1,3-thiazolidin-2-yliden)amino]-2phenylquinazolin-4(3H)-one (7f). Yield 58\%, m.p. $106^{\circ} \mathrm{C}$; IR $(\mathrm{KBr}) \mathrm{cm}^{-1}: 3025(\mathrm{C}-\mathrm{H}, \quad \mathrm{Ar}-\mathrm{H})$, 1382 (N-O), 1180 (C-O.); ${ }^{1} \mathrm{H}$ NMR (DMSO-d $\left.{ }_{6}\right): \delta 6.9-7.6(\mathrm{~m}, 16 \mathrm{H}, \mathrm{Ar}-\mathrm{H}), 5.9(\mathrm{~s}, 1 \mathrm{H},=\mathrm{CH}), 3.0$ (t, 2H, $\left.\mathrm{OCH}_{2}\right), 2.5$ (t, 2H, $\left.\mathrm{NCH}_{2}\right) ; \mathrm{MS}: \mathrm{m} / \mathrm{z}: 603\left[\mathrm{M}^{+}\right]$, 413, 382, 221, 189, 83. Anal. Calcd. for $\mathrm{C}_{32} \mathrm{H}_{21} \mathrm{~N}_{5} \mathrm{O}_{6} \mathrm{~S}:$ C, 63.68; H, 3.48; N, 11.60. Found : C, 63.50; H, 3.32; N, 11.52\%. 


\section{Acknowledgements}

The authors are thankful to the Head, Department of Chemistry, M. L. Sukhadia University, Udaipur (Rajasthan) for providing laboratory facilities and to the Director, CDRI, Lucknow for providing spectral and analytical data. Two of the authors (SO) and (DB) are thankful to CSIR and UGC, New Delhi respectively for providing financial assistance.

\section{References}

1. Kornet, M. J.; Waria, T.; Beaven, W. J. Heter.Chem. 1983, 20, 1553.

2. Alagarsamy, V.; Muthukumar, V.; Pavalarani, N.; Vasanthnathan, P.; Revathi, R. Biol. Pharm. Bull. 2003, 26, 557.

3. Alagarsamy, V.; Pathak, V. S.; Sriram, D.; Pandeya, S. N.; de Clercq, E. Ind. J. Pharm. Sci. 2000, 62, 433.

4. Abdel Ghaney, A. E. H.; Abdel waheb, M. H. Acta Pharm. 2003, 53, 127.

5. Pandey, V. K.; Tusi, S.; Tusi, Z., Raghubir, R.; Dixit, M.; Joshi, M. N.; Bajpai, S. K. Ind. J. Chem. 2004, 43B, 180.

6. Almasi, J.; Takacs-Novak, K.; Kokosi, J.; Varos, J. Int. J. Pharm. 1999, 180, 13.

7. Amir, M.; Khan, M. S. Y.; Zaman, M. S. Ind. J. Chem. 2004, 43B, 2189.

8. Hrib, N.; Jurcak, J.; Flavgare J. Med. Chem. 1992, 35, 2712.

9. Desyk, R. B.; Zimenkovsky, B. S. Current Org. Chem. 2004, B, 1547.

10. Biradar, J. S.; Manjunath, S. Y. Ind. J. Chem. 2004, 43B, 389.

11. Srivastava, V.; Singh, S.; Gulati, A.; Shankar, S. Ind. J. Chem. 1987, 26B, 652.

12. Loscher, W. G. Eur. J. Pharmacol. 1948, 342.

13. Bihzod, S.; Al-zaid, B.; Edafiogho, I. W. Seventh Annual Health Science Poster Day, 2002, p 108.

14. Berger, B. J. Antimicrob Agent Chemo. 2000, 44, 2540.

15. Banu, L.; Rajora; S.; Khatri, D; Talesara, G. L. J. Ind. Chem. Soc. 2000, 77, 300.

16. Singh, B.; Mehta, D.; Baregama, L. K. Ind. J. Chem. 2004, 43B, 1306.

17. Bauer, L.; Suresh, K. S. J. Org. Chem. 1963, 28, 1604. 